\title{
Effects of PCPA and selective REM sleep deprivation on rotorod performance and open-field behavior in the rat
}

\author{
C. RONALD CORUM \\ Institute of Physical Medicine and Rehabilitation, Louisville, Kentucky 40202 \\ and \\ JOHN B. THURMOND \\ University of Louisville, Louisville, Kentucky 40208
}

\begin{abstract}
The effects of P-chlorophenylalinine (PCPA) administration or of selective REM sleep deprivation (REMD) on open-field behavior and rotorod performance in rats were examined. Baseline performance and behavior were obtained over 10 days, then treatments were administered for 12 days, and recovery was measured over 5 days. Both PCPA and REMD produced shorter emergence latencies in the open field and increased locomotor activity and rearing. The effects of PCPA occurred during the first 6 days after injection, whereas the effects of REMD occurred during the last 6 days of the 12-day treatment period. Although PCPA and REMD produced similar changes in behavior, different mechanisms may have been involved.
\end{abstract}

Sleep research in the last decade has been aimed at elucidating the neurochemical mechanisms underlying sleep and waking (Holman, Elliot, \& Barchas, 1975). The monoamines, norepinephrine (NE) and serotonin (5-HT), are central to current theories on the regulation of sleep (Jouvet, 1972). NE may be involved in the initiation and regulation of wakefulness (Bartholini, Burkard, Pletscher, \& Bates, 1967; Moruzzi \& Magoun, 1959; Schaeppi \& Koella, 1966; Wyatt, Zarcone, Engelman, Dement, Snyder, \& Sjoerdsma, 1971), but the early notion that norepinephrine regulates REM sleep has not been supported (Hartmann, Chung, Draskocsy, \& Schildkraut, 1971; Henriksen \& Dement, 1972; King \& Jewett, 1971). The role of 5-HT in the regulation of REM sleep is not clear. Jouvet (1972) suggested that serotonergic neurons induce and maintain non-REM (NREM) sleep, and also trigger the onset of a REM sleep period. However, dorsal raphe units, which fire regularly during waking, slow down in NREM sleep and stop firing entirely in REM episodes (McGinty, Harper, \& Fairbanks, 1973). Whereas these findings question the degree to which 5-HT plays a role in regulating REM sleep, data from several studies suggest that similar behavioral changes follow 5-HT depletion and REM sleep deprivation. For example, median raphe lesions in rats lowered forebrain 5 -HT by $77 \%$ and increased running wheel and open-field activity as well as reactivity to novel stimuli and environmental change (Srebro \& Lorens, 1975). Similar behavioral changes have been reported in rats following REM sleep deprivation (Albert, Cicala, \& Siegel, 1970) and after administration of PCPA (Brody, 1970; Fibiger \&
Campbell, 1971), a well known specific depletor of 5-HT. We undertook the present investigation to compare behavioral changes following treatment with PCPA or selective REM sleep deprivation (REMD).

\section{METHOD}

Forty male COBS CD rats were used as subjects. They were 68 days old at the beginning of the experiment and weighed an average of $295 \mathrm{~g}$. Ten rats were assigned randomly to each of the four conditions of the experiment. The group administered PCPA and vehicle-injected controls were maintained individually in sawdust-bedded polyprophylene laboratory cages, $18 \times 28 \times 13.5 \mathrm{~cm}$. The rats deprived of REM sleep (REMD group) and their yoked controls (shock group) were housed individually in $25 \times 25 \times 25 \mathrm{~cm}$ cages in which the floor and sides were electrified with $50 \mathrm{~mA}$. The REMD rats avoided the electric grid by resting on round platforms provided by inverted flower pots, $7 \mathrm{~cm}$ in diameter and $13 \mathrm{~cm}$ high. The animals were given an adaptation period of 10 days before the experiment began, during which the environment was the same except that the round platform was $14 \mathrm{~cm}$ in diameter and $4 \mathrm{~cm}$ high. No platform was available to rats in the shock group, and consequently, they received brief shocks occasionally. These procedures were essentially identical to those used in prior studies (Cohen \& Dement, 1965 ; Joy \& Prinz, 1969), except that an electrified grid instead of water surrounded the flower pots. Food and water were available ad lib in all conditions of the study. A 12-h light cycle was imposed (on 7.00-19.00, off 19.00-7.00), and room temperature was constant at $25^{\circ} \mathrm{C}$.

The behavior of each group of animals was measured on each day of the experiment at $10.00 \mathrm{~h}$ using rotorod (Kinnard \& Carr, 1957) and open-field procedures (Brimblecombe, 1963). The open field was $68.5 \mathrm{~cm}$ high and $122 \mathrm{~cm}$ square, marked off into 16 equal squares. The interior of the apparatus was white with black lines separating the floor into the divisions. A single $100-\mathrm{W}$ bulb illuminated the field from a distance of $26 \mathrm{~cm}$ above the 
center. During testing, the rats were placed in a $28 \times 28 \times 18 \mathrm{~cm}$ wire cage, lowered gently into a corner square of the open field, and observed for $2 \mathrm{~min}$. Data obtained consisted of emergence latencies from this "home square," transversement of the perimeter squares, center squares entered, number of rearings, and stool count. Immediately afterward, performance on the rotorod was assessed by placing the rat on a $4-\mathrm{cm}$ wooden dowel that revolved at $11 \mathrm{rpm}$. Five trials were given, each lasting a maximum of $2 \mathrm{~min}$, during which the number of falls and the latency to fall were recorded. Each rat was given practice using this procedure on each of 10 days of the adaptation period, which preceded the experimental conditions.

Following the period of adaptation, the experiment was divided into three phases: Phase 1 , in which the rats were permitted to sleep normally for 10 days (the REMD group on large inverted flower pots, others in cages); Phase 2, in which for 12 days experimental conditions (controls, PCPA, REMD, and shock groups) were instituted; and Phase 3, in which the rats were given a 5 -day recovery period. At the beginning of Phase 2, an i.p. injection of PCPA $(400 \mathrm{mg} / \mathrm{kg})$ methyl ester was given to the PCPA group, and PCPA was added to the drinking water of each rat at a concentration of $.5 \mathrm{mg} / \mathrm{ml}$. Likewise, at the beginning of Phase 2 , the control group of animals received an i.p. saline injection, adjusted for $\mathrm{pH}$ to match that of the PCPA solution. On the first day of Phase 2, the amount of drinking water containing PCPA consumed by the rats dropped from the baseline level of $35 \mathrm{ml}$ to approximately $15 \mathrm{ml}$, but this returned to baseline on the following day and varied about the baseline level for the remainder of the study (about $25-50 \mathrm{mg} / \mathrm{kg}$ of PCPA daily). The shock group of rats were yoked to the REMD rats in such a manner that a rat was shocked each time a REMD counterpart fell from the pot until the time that he regained his position on the top of the pot. Statistical comparisons for the evaluation of differences in rotorod performance between the experimental groups and their appropriate controls were based on the $t$ test (two-tailed) for independent measures. Changes in open-field behavior as a function of the various treatments were evaluated by comparisons with baseline levels using the t test (two-tailed) for correlated measures.

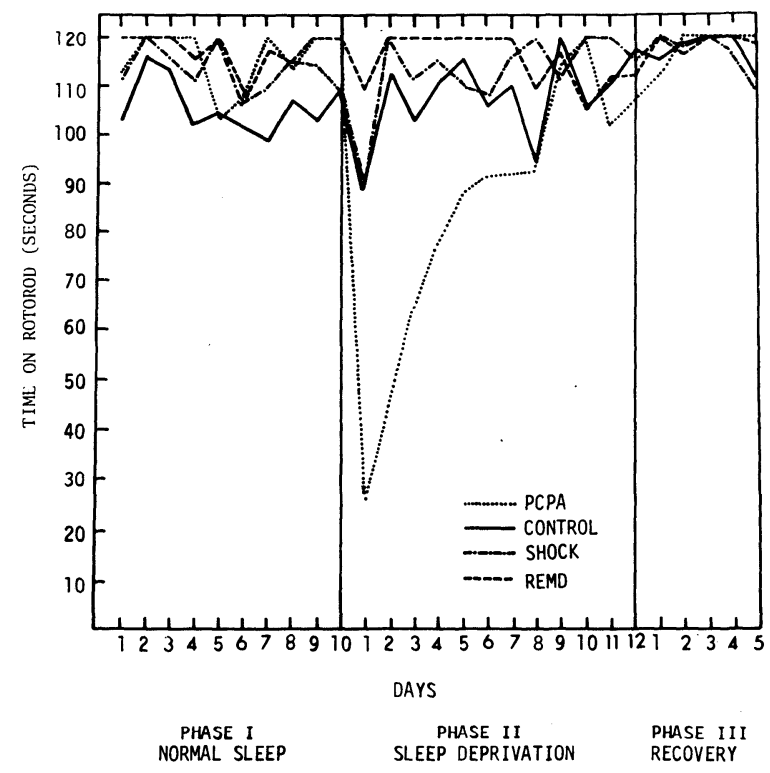

Figure 1. Comparison of the effects of PCPA and sleep deprivation (REMD) on the number of seconds the animals stayed on the rotorod before falling off. Each point represents the mean of 10 animals.

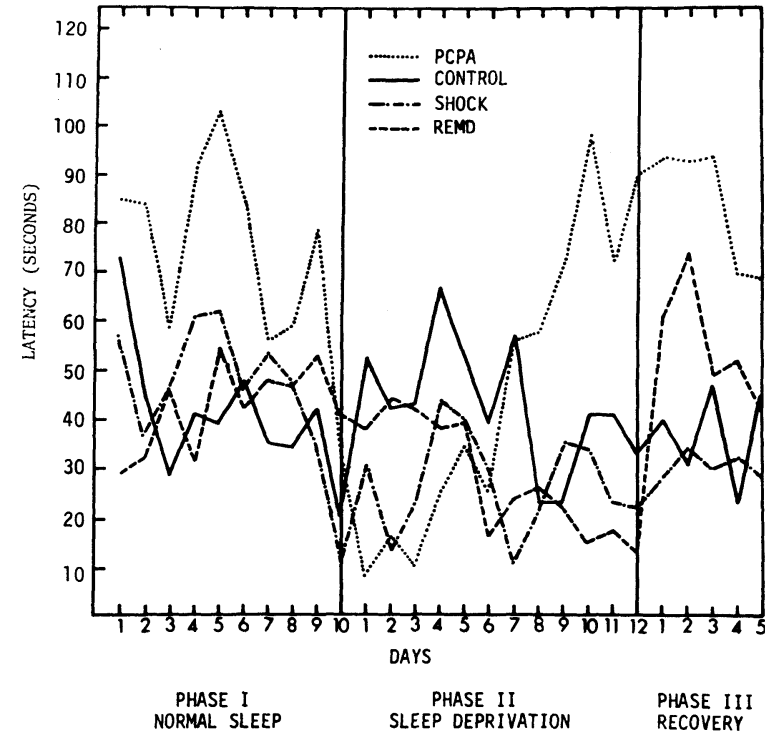

Figure 2. Comparison of PCPA and REMD effects on emergence latency from the home square of the open field. Each point represents the mean of 10 animals.

\section{RESULTS}

Figure 1 shows that the rotorod performance dropped markedly following the injection of PCPA at the beginning of Phase 2, but then continued to improve despite administration of the drug in the drinking water. Significant changes in rotorod performance of the other groups throughout the study did not occur. The rotorod performance of the PCPA group during the first half of Phase 2 differed significantly from that of the control group during this same period $[\mathrm{t}(18)=3.87, \mathrm{p}<.005]$.

To compare the effects of PCPA treatment and selective REM sleep deprivation on open-field behavior (Figures 2, 3, and 4), we averaged the measures obtained over the last 6 days of Phase 1 (normal sleep) and compared to these the behavior of each group during the first half of Phase 2 (6 days) and the last half of Phase 2 (6 days).

In Figure 2 is shown the emergence latency in the open field. The PCPA group emerged with significantly shorter latencies during the first half of Phase 2 $[t(9)=4.72, p<.002]$. This behavior contrasted with that of the animals that displayed a continuous drop in performance throughout Phase 2, reaching a low point on the last day of sleep deprivation (Day 12). Compared to the baseline level for the REMD rats, the shorter latency over the last half of Phase 2 is significantly different $[t(9)=2.35, p<.005]$. By the time PCPA administration was discontinued at the beginning of Phase 3 (recovery), the PCPA group was emerging with latencies slightly longer than their baseline levels. In the REMD group, a rebound effect was apparent at the onset of recovery during the first 2 days of Phase 3 ; the sudden increase in latencies over these 2 days, com- 

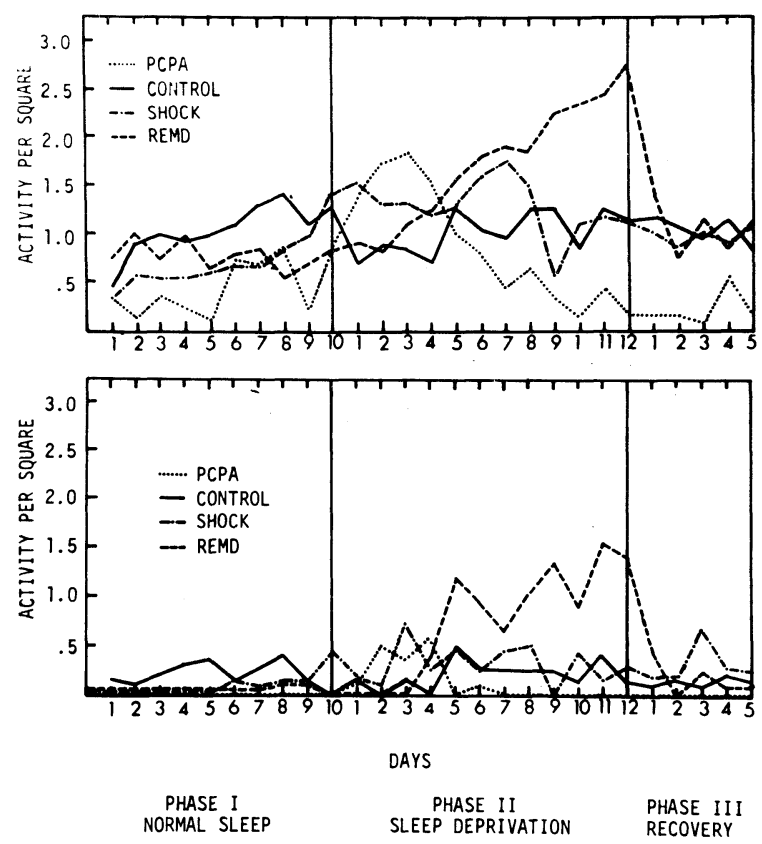

Figure 3. Comparison of PCPA and REMD effects on activity in the perimeter of the open field (top graph) and in the center of the field (bottom graph). Each point represents the mean of 10 animals.

pared with the last 2 days of baseline, was significant $[t(9)=2.65, p<.05]$.

A comparison of activity in the open field is presented in Figure 3, the locomotion being shown separately for the perimeter and the center of the field. The PCPA group's increase in activity during the first half of Phase 2 was significantly higher than baseline in the perimeter $[t(9)=3.78, p<.005]$ and in the center squares $[t(9)=3.56, p<.01]$. Similar significant increases in activity for the REMD group occurred during the last half of Phase 2 for the perimeter $[\mathrm{t}(9)=5.39, \mathrm{p}<.001]$ and center squares $[\mathrm{t}(9)=2.84$, $\mathrm{p}<.02]$.

The number of rearings made in the open field (Figure 4) showed changes during Phase 2, which were essentially the same as those obtained for locomotor activity. The PCPA group displayed a significant increase in rearings during the first half of Phase $2[\mathrm{t}(9)=2.41$, $\mathrm{p}<.05]$, whereas the REMD group's increase in rearings occurred during the last half of Phase $2[\mathrm{t}(9)=3.47$, $\mathrm{p}<.01]$.

Finally, a count of the number of stools deposited by each animal was made during the study. The PCPA group did not differ from the control group on this measure. However, compared to their baseline levels over the last 6 days of Phase 1, the stool count decreased significantly for the REMD group during the last 6 days of sleep deprivation in Phase $2[\mathrm{t}(9)=4.08, \mathrm{p}<.005]$, as it did for their yoked controls in the shock group during the same period $[\mathrm{t}(9)=2.86, \mathrm{p}<.02]$.

\section{DISCUSSION}

Except for a slight and nonsignificant drop in performance on the rotorod following the vehicle injection given at the conclusion of baseline data collection, the control group's performance on the rotorod and their behavior in the open field changed little throughout the study. The stress of the periodic shock that the yoked control animals received did not affect their rotorod performance, but this group did show a reduction in defecation bearing out Broadhurst's (1957) conclusion that shock reduced defecation.

The differences in behavior between the PCPA group and the REMD group during Phase 2 relate principally to the time at which the changes in behavior occurred in the two groups. Perhaps the most striking difference between the two groups occurred with respect to their performance on the rotorod at the beginning of Phase 2, that is, at the beginning of PCPA administration to the PCPA group and selective REM sleep deprivation in the REMD group. The initial precipitous drop in rotorod performance in the PCPA group can probably be attributed to a neurotoxic effect of the drug. Similar neurotoxic effects of PCPA on rotorod performance in mice have been reported recently by Rolinski (1975). The rotorod was chosen to reveal motor deficits since locomotor coordination is needed to maintain position on the rotorod. The time course of recovery from the effects of the PCPA injection is in agreement with the finding of Fibiger and Campbell (1971) that the time course of increased activity measured by a stabilimeter following PCPA intubation was of 6 days duration. Also, these investigators observed that during a time course of 12 days in a running wheel following administration of PCPA, the animals exhibited an initial depression of activity during the first $24 \mathrm{~h}$, followed by a biphasic increase and decrease in activity.

The PCPA group and the REMD group displayed essentially identical changes in open-field behaviors compared to their baseline levels, the difference being that the PCPA induced these changes quickly, followed by recovery toward baseline, whereas selective REM sleep deprivation had a cumulative effect that showed up later during the course of Phase 2. Thus, soon after administration of PCPA was initiated at the beginning of Phase 2, and near the end of Phase 2 after the cumulative effects of REM deprivation occurred, both the PCPA and the REMD groups displayed decreased emergence latencies, increased perimeter and center activity, and an increased number of rearings. Similar effects of PCPA on open-field behavior have been obtained in prior studies (Brody, 1970). Fibiger and Campbell (1971) have suggested that this heightened behavioral arousal may be due to a decrease in arousal thresholds following PCPA administration.

Our results indicating that selective REM sleep deprivation leads to increased activity in the open field are in agreement with

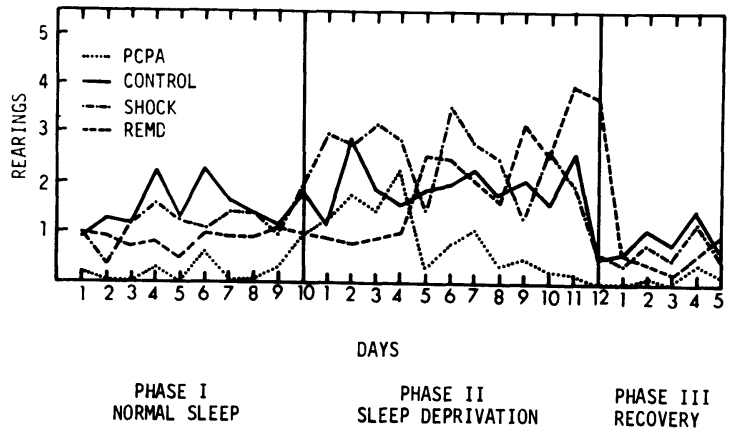

Figure 4. Comparison of PCPA and REMD effects on the number of rearings in the open field. Each point represents the mean of 10 animals. 
those of a prior study in rats (Albert et al., 1970). Thus, there is an apparent similarity between the behavioral effects observed after treatment with PCPA and REM sleep deprivation. However, studies relating the effects of PCPA to sleep deprivation have failed to show a clear relation between the two variables. Rechtshaffen, Lovell, Freedman, Whitehead, and Aldrich (1973) were unable to demonstrate significant changes in sleep-waking with PCPA treatment in rats, and a similar failure has occurred in humans (Knapp \& Mandell, 1972). Dement, Mitler, and Henriksen (1972) reported that chronic PCPA administration in cats produced only a transient insomnia lasting 3 to 4 days, despite continued marked cerebral serotonin depletion. These data suggest that different mechanisms may be responsible for the similar behavioral changes observed.

\section{REFERENCES}

Albert, I., Cicala, G. A., \& Siegel, J. The behavioral effects of REM sleep deprivation in rats. Psychophysiology, $1970,6,550-560$.

Bartholin, G., Burkard, W. P., Pletscher, W. P., \& BATES, H. M. Increase of cerebral catecholamines caused by 3,4 dehydroxyphenylalanine after inhibition of peripheral decarboxylase. Nature, 1967, 215, 852-853.

BRIMBLECOMBE, R. W. Effects of psychotropic drugs on open field behavior in rats. Psychopharmacologia, 1963, 4, 139-147.

BroaDhuRst, P. L. Determinants of emotionality in the rat, I situational factors. British Journal of Psychology, 1957, 48, 1-12.

BRODY, J. F. Behavioral effects of serotonin depletion and p-chlorophenylalanine (a serotonin depletor) in rats. Psychopharmacologia, 1970, 17, 14-33.

Cohen, H., \& Dement, W. Sleep: Changes in threshold to electroconvulsive shock in rats after deprivation of "paradoxical sleep." Science, 1965, 150, 1318-1319.

Dement, W. C., Mitler, M. M., \& Henriksen, S. J. Sleep changes during chronic administration of parachlorophenylalanine. Review of Canadian Biology, 1972, 31, 239-246.

Fibiger, H. C., \& Camprell, B. A. The effect of parachlorophenylalanine on spontaneous locomotor activity in the rat. Neuropharmacology, 1971, 10, 25-32.

Hartmann, E., Chung, R., Draskocsy, P. R., \& SCHILDKRAUT, J. J. Effects of 6-Hydroxydopamine on sleep in the rat. Nature, 1971, 233, 425-427.

Henriksen, S., \& Dement, W. In M. Chase, W. Stern, \& P. Walter (Eds.), Sleep research. Los Angeles: BIS/BRI, 1972. P. 55.

Holman, R., Elliot, G., \& Barchas, J. Neuroregulators and sleep mechanisms. Annual Review of Medicine, 1975, 26, 499-520.
Jouvet, M. The role of monoamines and acetylocholinecontaining neurons in the regulation of the sleep-waking cycle. Ergebnisse der physiologie, 1972, 64, 166-307.

Joy, R. M., \& Prinz, P. N. The effect of sleep altering environments upon the acquisition and retention of a conditioned avoidance response in the rat. Physiology and Behavior, 1969, 4, 809-814.

King, D., \& JEWETT, R. E. The effects of a-methytyrosine on sleep and brain norepinephrine in cats. Journal of Pharmacology and Experimental Therapeutics, 1971, 177, 188-194.

Kinnard, W. J., \& CARR, C. J. A preliminary procedure for the evaluation of central nervous system depressants. Journal of Pharmacology and Experimental Therapeutics, 1957, 114, 354-361.

KnapP, S., \& Mandell, A. J. PCPA-it's three phase sequence of interaction with two forms of brain tryptrophan hydroxylase. Life Science, 1972, 11, 761-771.

McGinty, D. J., Harper, R. M., \& FairbanKs, M. K. 5-HT-containing neurons: Unit activity in behaving cats. In J. Barchas \& E. Usdin (Eds.), Serotonin and behavior. New York: Academic Press, 1973. Pp. 267-280.

MoruzzI, G., \& Magoun, H. W. Brain stem reticular formation and activation of the EEG. Electroencephalography and Clinical Neurophysiology, 1949, 1, 455-473.

RechtschafFen, A., LOVEll, R. A., Freedman, D. X., Whitehead, P. K., \& Aldrich, M. The effect of parachlorophenylaline on sleep in the rat: Some implications for the serotonin-sleep hypothesis. In J. Barchas \& E. Usdin (Eds.), Serotonin and behavior. New York: Academic Press, 1973. Pp. 401-418.

RolinskI, Z. Pharmacological studies on isolation-induced aggressiveness in mice in relation to biogenic amines. Polish Journal of Pharmacology and Pharmacy, 1975, 27, 37-44.

Schaeppi, U., \& Koella, W. P. Changes in level of vigilance in response to administration of drugs into the 4th ventricle of the rat. Federation Proceeding, 1966, 25, 628.

SREBro, B., \& Lorens, S. A. Behavioral effects of selective midbrain Raphe lesion in the rat. Brain Research, 1975, 89, 303-325.

Wyatt, R. J., Zarcone, V., Engelman, K., Dement, W. C., SNYDER, S., \& SJOERDSMA, A. Effects of 5-hydroxytryptophan on the sleep of normal human subjects. Electroencephalography and Clinical Neurophysiology, 1971, 30, 505-509.

(Received for publication January 10, 1978.) 